\title{
Varying Dose of Atropine in Slowing Myopia Progression in Children Over Different Follow-Up Periods by Meta-Analysis
}

\begin{abstract}
Jiahe Gan 1,2, Shi-Ming $\mathrm{Li}^{1,2 *}$, Shanshan $\mathrm{Wu}^{3}$, Kai Cao ${ }^{1,2}$, Dandan Ma ${ }^{1,2}, \mathrm{Xi} \mathrm{He}^{1,2}$, Ziyu Hua ${ }^{1,2}$, Meng-Tian Kang ${ }^{1,2}$, Shifei Wei ${ }^{1,2}$, Weiling Bai ${ }^{1,2}$ and Ningli Wang ${ }^{1,2 *}$

${ }^{1}$ Beijing Tongren Eye Center, Beijing Institute of Ophthalmology, Beijing Tongren Hospital, Capital Medical University, Beijing, China, ${ }^{2}$ Beijing Ophthalmology and Visual Science Key Laboratory, Beijing Tongren Eye Center, Beijing Tongren Hospital, Capital Medical University, Beijing, China, ${ }^{3}$ Department of Epidemiology and Health Statistics, Peking University School of Public Health, Beijing, China
\end{abstract}

OPEN ACCESS

Edited by:

Michele Lanza

University of Campania Luigi

Vanvitelli, Italy

Reviewed by:

Hou-Wen Lin,

Shanghai Jiao Tong University, China

Xuejuan Jiang,

University of Southern California,

United States

Zilu Zhang,

Harvard Medical School,

United States

Jirong Yue,

Sichuan University, China

${ }^{*}$ Correspondence:

Ningli Wang

wningli@vip.163.com

Shi-Ming L

lishiming81@163.com

Specialty section:

This article was submitted to

Ophthalmology,

a section of the journal

Frontiers in Medicine

Received: 10 August 2021 Accepted: 13 December 2021 Published: 13 January 2022

Citation:

Gan J, Li S-M, Wu S, Cao K, Ma D, He X, Hua Z, Kang M-T, Wei S, Bai W and Wang N (2022) Varying Dose of

Atropine in Slowing Myopia Progression in Children Over Different Follow-Up Periods by Meta-Analysis.

Front. Med. 8:756398.

doi: 10.3389/fmed.2021.756398
Purpose: To evaluate the efficacy and safety of atropine for slowing myopia progression and to investigate whether the treatment effect remains constant with continuing treatment.

Method: Studies were retrieved from MEDLINE, EMBASE, and the Cochrane Library from their inception to May 2021, and the language was limited to English. Randomized controlled trials (RCTs) and cohort studies involving atropine in at least one intervention and placebo/non-atropine treatment in another as the control were included and subgroup analysis based on low dose $(0.01 \%)$, moderate dose $(0.01 \%-<0.5 \%)$, and high dose (0.5-1.0\%) were conducted. The Cochrane Collaboration and Newcastle-Ottawa Scale were used to evaluate the quality of RCTs and cohort studies, respectively.

Results: Twelve RCTs and fifteen cohort studies involving 5,069 children aged 5 to 15 years were included. The weighted mean differences in myopia progression between the atropine and control groups were 0.73 diopters $(\mathrm{D}), 0.67 \mathrm{D}$, and $0.35 \mathrm{D}$ per year for high-dose, moderate-dose, and low-dose atropine, respectively $\left(\chi^{2}=13.76 ; P=\right.$ $\left.0.001, R^{2}=85.5 \%\right)$. After removing studies that provided extreme findings, atropine demonstrated a significant dose-dependent effect on both refractive change and axial elongation, with higher dosages of atropine resulting in less myopia progression $(r=0.85$; $P=0.004)$ and less axial elongation $(r=-0.94 ; P=0.005)$. Low-dose atropine showed less myopia progression $(-0.23 \mathrm{D} ; P=0.005)$ and less axial elongation $(0.09 \mathrm{~mm}, P$ $<0.001)$ in the second year than in the first year, whereas in high-dose atropine more axial elongation $(-0.15 \mathrm{~mm}, P=0.003)$ was observed. The higher dose of atropine was associated with a higher incidence of adverse effects, such as photophobia with an odds ratio (OR) of 163.57, compared with an OR of 6.04 for low-dose atropine and 8.63 for moderate-dose atropine $(P=0.03)$.

Conclusion: Both the efficacy and adverse effects of atropine are dose-dependent in slowing myopia progression in children. The efficacy of high-dose atropine was reduced after the first year of treatment, whereas low-dose atropine had better efficacy in a longer follow-up period.

Keywords: atropine, myopia, efficacy \& safety, dose, follow-up 


\section{INTRODUCTION}

Myopia has emerged as a serious public health issue with a rapidly increasing prevalence worldwide $(1,2)$, especially in some Asian areas (3-6). The myopia prevalence reached $52.7 \%$ in 2020 among Chinese adolescents, which prompted Chinese governments to implement nationwide myopia control policies including increasing the engagement of children in outdoor activities. However, the deadly outbreak of the coronavirus disease 2019 (COVID-19) pandemic largely reduced opportunities for children to spend time outdoors (7). Prolonged home confinement has brought excessive time for near work and insufficient time outdoors, both of which have been recognized as major environmental risk factors for myopia development (8-10).

Therefore, solutions for myopia management are of great social concern. In recent years, the treatment with different doses of topical atropine has been recognized as currently one of the most effective treatments for myopia (11), and has been applied to more than $60 \%$ of children with myopia in Taiwan (12). However, it is still pending approval by the FDA and has remained an off-label treatment in mainland China and most of the western countries since high doses $(0.5-1 \%)$ of atropine have inevitable ocular side effects, such as cycloplegia, photophobia, allergic reaction, blurred near vision, and accelerated progression on cessation (rebound effect) $(13,14)$. Therefore, moderate doses (0.01-0.5\%) and low doses of atropine $(0.01 \%)$ have been widely applied in clinical treatment for children with myopia in recent years.

In our previous meta-analysis, we found a difference in efficacy of atropine among different ethnicities, with greater effects in Asians than in white children (15). Then, we conducted the first randomized clinical trials (RCTs) on low-dose atropine in mainland China and found a $34.2 \%$ reduction in myopia progression within 1 year (16). However, there are still some uncertainties and controversies. Some studies reported that the efficacy of atropine was dose-related (17), whereas others found that efficacy of atropine was dose-independent within the range of $0.01-1 \%(13,18)$. Most RCTs and cohort studies reported a first-year protective effect on myopia, whereas the Atropine for the Treatment of Myopia 2 (ATOM 2) study showed a better effect of $0.01 \%$ atropine treatment in the second year than in the first year, and it is recommended that the initial treatment of $0.01 \%$ atropine should last at least 2 years (19). But the evidence is still lacking on whether continuing eyedrops for a longer duration of treatment can produce a continued effect $(20,21)$. In addition, some eye-care professionals have been concerned that potential side effects (e.g., photophobia) of atropine may affect children's quality of life and reduce compliance, which may influence the efficacy of myopia control. Therefore, an optimal dose of atropine with substantial efficacy and acceptable side effects has remained undetermined. Comparison of different doses is essential to enable clinicians and parents to choose the safest and most effective treatment for myopia control.

In this meta-analysis, we aimed to evaluate the overall efficacy and safety of different doses of atropine with more updated RCTs and cohort studies and to explore the dose-response relationship of atropine. We also investigated whether there was an efficacy difference across different treatment periods.

\section{METHODS}

This meta-analysis was performed in compliance with the Preferred Reporting Items for Systematic Reviews and Meta-Analyses (PRISMA) guidelines (eTable 1 in the Supplementary Material) (22).

\section{Eligibility Criteria}

We included comparative studies (i.e., RCTs, and cohort studies) according to the following criteria: (1) a human study investigating the relationship between topical atropine and myopia in school-aged children (between 6 and 15 years); (2) using atropine in at least one intervention and placebo or nonatropine treatment in another as the control; and (3) reporting at least one outcome of interest, including the annual rate of myopia progression and any adverse effects. In addition, the dose of atropine was classified into 3 subgroups: low dose $(0.01 \%)$, moderate dose $(>0.01 \%$ to $<0.5 \%)$, and high dose (0.5-1.0\%) (23).

\section{Search Methods}

Data were obtained from MEDLINE, EMBASE, and the Cochrane Library from their inception to May 2021 with language striction in English. We selected RCTs and cohort studies involving atropine in at least one treatment arm and placebo or non-atropine treatment in another as the control that reported myopia progression and/or side effects of atropine therapy for analysis. Medical Subject Headings (MeSH) and the following as keywords: myopia, refractive errors, muscarinic antagonists, cholinergic antagonists, mydriatics, atropine, clinical trial, and humans, as well as some relevant free terms were used for search. Boolean operators "AND," "OR," "NOT" were used to combine all search sets. Detailed search strategies are provided in eTable 2 in the Supplementary Material. We also screened clinicaltrials.gov and the reference lists of published reviews to identify additional relevant studies. Exclusion criteria were (at least one of the following): overlapping population; nonhuman studies; lack of data for outcomes of interest; and studies published as abstracts, reviews, case reports, comments, letters to the editor, and animal research.

\section{Data Extraction and Quality Assessment}

Two investigators (GJH and MDD) independently screened the titles, abstracts, and full-text articles for inclusion using standardized data extraction forms. When the same population was involved in multiple reports, only the latest report was included to avoid duplicated data. Both investigators extracted the study characteristics from each trial: (1) first author, (2) year of publication, (3) study design, (4) country or area, (5) intervention and control, (6) follow-up duration, (7) sample size, (8) baseline characteristics (sex, age, refraction, axial length, dropouts from total number), (9) endpoints (mean change in refraction and axial length), and (10) number of side effects. All disagreements were reviewed by a third investigator (HX). 
For any missing data, we contacted the authors of the trial reports or used GetData GraphDigitizer 2.24 (http://getdatagraph-digitizer.com) to read data from figures. The list of exclusion studies and reasons for exclusion were shown in eTable 3 in the Supplementary Material. The quality of the selected trials was assessed by the following six aspects following the recommendations of Cochrane collaboration (24) for RCTs: allocation sequence generation, allocation concealment, masking of patients and clinicians, masking of outcome assessors, incomplete outcome data, and selective outcome reporting. Newcastle-Ottawa Quality Assessment Scale items (25) with a "star system" were applied to assess the quality of cohort studies and included 8 items within 3 domains: selection (representativeness), comparability (because of design or analysis), and outcomes (assessment and follow-up). A study can be awarded a maximum of 1 star for each numbered item within the selection and outcome categories and a maximum of 2 stars can be given for comparability, and the total scores range from 0 to 9 stars. Stars of $0-3,4-6,7-9$ were considered as low, moderate, and high quality, respectively (26).

\section{Outcome}

The efficacy outcome were mean annual changes in refraction [diopters (D)/year], axial length ( $\mathrm{mm} /$ year), and the number of children showing myopia progression. The safety outcomes were the number of adverse events including photophobia, blurred near vision, and allergy. We also extracted data on photopic and mesopic pupil diameter $(\mathrm{mm})$ and change in accommodation (amplitude/year).

\section{Statistical Analysis}

Data analyses were conducted using Review Manager (Version 5.4; The Cochrane Collaboration, 2020). We calculated the weighted mean difference (MD) and 95\% confidence intervals (95\% CIs) for different doses of atropine in refractive changes and axial elongation vs. the control group, as well as the odds ratios (ORs) for adverse effects between the atropine and control groups. The effect sizes (ESs) were calculated using the Cohen d formula. ORs with 95\% CIs of proportions with fast $(>1.0$ diopters (D) per year)/slow ( $<0.5 \mathrm{D} /$ year) myopia progression was also calculated. Heterogeneity was assessed using Cochran's $Q$-test and $I^{2}$ statistics. If the heterogeneity was not significant ( $p$ $>0.1, I^{2}<50.0 \%$ ), a fixed-effects model was used; otherwise, a random-effects model was used.

Sensitivity analysis was performed by excluding studies with significantly different characteristics to assess their influence on the overall estimates. Subgroup analyses were pre-planned to compare the treatment effects among children with different doses of atropine [low dose $(0.01 \%)$, moderate dose $(>0.01$ to $<0.5 \%)$, high dose $(0.5-1.0 \%)]$, treatments in control groups (placebo or non-placebo), and ethnicity. Meta-regression analysis was also conducted to identify potential sources of heterogeneity. $P$ for interaction was performed using linear mixed effects model, where we built a product term of doses of atropine $\times$ ethnicity, as well as a product term of doses of atropine $\times$ study design (RCT or cohort study). Publication bias was assessed by visual inspection of funnel plot if the number of retrieved studies

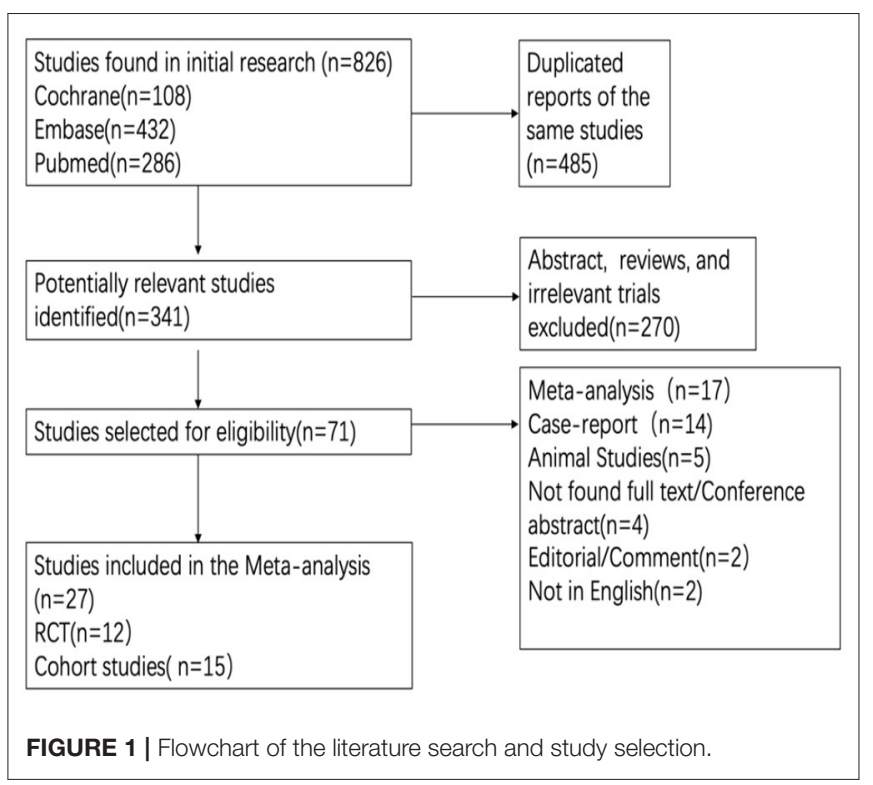

was $>10 . P<0.05$ was considered statistically significant for all analyses.

\section{RESULTS}

The search yielded a total of 826 articles, of which 12 RCTs $(16,27-36)$ and 15 cohort studies (37-51) were included for final analysis (Figure 1). Table 1 details the relevant features of the 27 studies. Briefly, the total sample size of participants included in our study was 5,069, among which 3,024 were received atropine treatment and 2,045 participants were received placebo or non-atropine treatment, with a follow-up period from 12 to 144 months. Concerning geographical location of the studies, 7 studies were conducted in mainland China, 8 in Taiwan, 4 in the United States, 2 in Singapore, 2 in Hong Kong, 2 in Europe, 1 in Japan, and 1 in India, resulting in most participants being Asian. All RCTs were conducted in Asia, among which Wei et al. (16) provided the first placebo-controlled RCT data for low-dose atropine in mainland China.

\section{Risk of Bias Assessment}

Bias for the included RCTs is presented in eTable 4 in the Supplementary Material. There were two RCTs $(30,35)$ assessed as high risk of bias due to unclear randomization, inadequate loss to follow-up and without blinding. The quality of the included cohort studies was generally high according to the Newcastle-Ottawa Scale items (26) (eTable 5 in the Supplementary Material).

\section{Effect of Atropine on the Annual Rate of Myopia Progression}

Changes in refraction from 12 RCTs and 15 cohort studies were obtained. Since no difference between RCTs and cohort studies was observed in low-dose, moderate-dose, and high-dose subgroups (eFigure 1 in the Supplementary Material; all $P>$ 
TABLE 1 | Characteristics of the studies included in the meta-analysis.

\begin{tabular}{|c|c|c|c|c|c|c|c|}
\hline \multirow[t]{2}{*}{ References } & \multirow[t]{2}{*}{ Country/Area } & \multirow{2}{*}{$\begin{array}{l}\text { Study } \\
\text { design }\end{array}$} & \multirow{2}{*}{$\begin{array}{l}\text { Follow-up } \\
\text { (months) }\end{array}$} & \multirow{2}{*}{$\begin{array}{c}\text { Age } \\
\text { (years) }\end{array}$} & \multicolumn{2}{|c|}{ Intervention } & \multirow{2}{*}{$\begin{array}{c}\text { Baseline } \\
\text { refraction, } \\
\text { diopter mean } \\
\text { (SD) }\end{array}$} \\
\hline & & & & & $\begin{array}{l}\text { Experimental group } \\
\text { (atropine dose, \%) }\end{array}$ & Control group & \\
\hline Yen et al. (35) & Taiwan, China & $\mathrm{RCT}$ & 12 & $6-14$ & 1.00 every other night & Placebo & $-1.52 \pm 0.92$ \\
\hline \multirow[t]{3}{*}{ Shih et al. (30) } & Taiwan, China & $\mathrm{RCT}$ & 24 & $6-13$ & $0.5 \%+$ bifocals & $\begin{array}{l}0.5 \% \text { tropicamide } \\
\text { nightly + full } \\
\text { correction }\end{array}$ & $-4.41 \pm 1.47$ \\
\hline & & & & & $\begin{array}{l}0.25 \%+\text { partially } \\
\text { undercorrected glasses }\end{array}$ & & \\
\hline & & & & & $\begin{array}{l}0.1 \%+\text { full eyeglass } \\
\text { correction }\end{array}$ & & \\
\hline Shih et al. (31) & Taiwan, China & $\mathrm{RCT}$ & 18 & $6-13$ & $0.5 \%+$ multifocal lenses & Multifocal lenses & $-3.26 \pm 0.15$ \\
\hline Chua et al. (27) & Singapore & $\mathrm{RCT}$ & 24 & $7-12$ & 1.00 & Placebo & $-3.36 \pm 1.38$ \\
\hline Chia et al. (28) & Singapore & $\mathrm{RCT}$ & 48 & $6-12$ & $0.5,0.1,0.01$ & - & $0.38 \pm 0.60$ \\
\hline Yi et al. (36) & China & $\mathrm{RCT}$ & 12 & $6-12$ & 1.00 & Placebo & $-1.23 \pm 0.32$ \\
\hline Wang et al. (32) & China & $\mathrm{RCT}$ & 12 & $5-10$ & 0.50 & Placebo & $-1.30 \pm 0.40$ \\
\hline Yam et al. (34) & China & $\mathrm{RCT}$ & 12 & $4-12$ & $0.05,0.025,0.01$ & Placebo & -1.00 or less \\
\hline Wei et al. (16) & China & RCT & 12 & $6-12$ & 0.01 & Placebo & $-2.52 \pm 1.33$ \\
\hline Zhu et al. (33) & China & $\mathrm{RCT}$ & 48 & $6-12$ & 1 & Placebo & $-3.82 \pm 0.44$ \\
\hline Saxena et al. (52) & India & $\mathrm{RCT}$ & 12 & $6-14$ & 0.01 & Placebo & $-3.5 \pm 1.3$ \\
\hline Hieda et al. (53) & Japan & $\mathrm{RCT}$ & 24 & $6-12$ & 0.01 & Placebo & -1.00 to -6.00 \\
\hline Bedrossian (37) & USA & Cohort & 33 & $8-12$ & 1 & Blank & -0.50 or less \\
\hline Chou et al. (38) & Taiwan, China & Cohort & 38 & $7-14$ & 0.5 & Self-contrast & -6.25 to -12.00 \\
\hline Kennedy et al. (45) & USA & Cohort & 144 & $6-15$ & 1 & Blank & -1.49 \\
\hline Lee et al. (40) & Taiwan, China & Cohort & 20 & $6-12$ & 0.05 & Blank & $-1.58 \pm 1.37$ \\
\hline Fan et al. (39) & Hongkong, China & Cohort & 12 & $5-10$ & 1 & Blank & $-5.18 \pm 2.05$ \\
\hline Fang et al. (43) & Taiwan, China & Cohort & 18 & $6-12$ & 0.025 & Blank & $-0.31 \pm 0.45$ \\
\hline Wu et al. (50) & Taiwan, China & Cohort & 54 & $6-12$ & 0.05 & Blank & $-2.45 \pm 1.63$ \\
\hline Lin et al. (41) & China & Cohort & 12 & $8-15$ & 1.00 & Self-contrast & $-1.92 \pm 0.91$ \\
\hline Clark and Clark (42) & USA & Cohort & 13 & $6-15$ & 0.01 & Blank & $-2.00 \pm 1.60$ \\
\hline Lee et al. (46) & Taiwan, China & Cohort & 12 & $5-14$ & $0.125,0.25$ & Blank & $-1.45 \pm 0.69$ \\
\hline Polling et al. (54) & Europe & Cohort & 12 & $8-13$ & 0.50 & $\begin{array}{l}\text { Withdraw } \\
\text { population }\end{array}$ & $-6.70 \pm 3.60$ \\
\hline Moon and Shin (44) & Korea & Cohort & 12 & $5-14$ & $0.01,0.025,0.05$ & Self-contrast & $-3.84 \pm 2.47$ \\
\hline Larkin et al. (47) & USA & Cohort & 24 & $6-15$ & 0.01 & Blank & $-3.10 \pm 1.90$ \\
\hline Sacchi et al. (49) & Europe & Cohort & 12 & $5-14$ & 0.01 & Blank & $-3.00 \pm 2.23$ \\
\hline Fu et al. (51) & China & Cohort & 12 & $6-12$ & $0.01,0.02$ & Blank & $-2.76 \pm 1.47$ \\
\hline
\end{tabular}

0.05 in the test for subgroup difference), we thus evaluated the effects of atropine by combining RCTs and cohort studies to provide larger samples for different doses.

The pooled data revealed significantly less progression in refraction for low-dose (MD, $0.35 \mathrm{D}$ per year; $95 \% \mathrm{CI}, 0.22-0.48 \mathrm{D}$ per year; $P<0.001$ ), moderate-dose (MD, 0.67D per year; $95 \%$ CI, 0.31-1.03D per year; $P<0.001$ ), and high-dose (MD, 0.73D per year; 95\% CI, 0.57-0.98D per year; $P<0.001$ ) atropine groups than control groups (Figure 2 ). There was a statistically significant difference in refraction changes among various doses of atropine within this range $\left(\chi^{2}=13.76 ; P=0.001\right.$ for subgroup difference; $I^{2}=85.5 \%$ ). The effect sizes showed a large treatment effect in different dose atropine groups (Figure 3). We observed no correlation between a dose and treatment effect $(r=0.665 ; P$ $=0.051)$. However, when the study by Moon and Shin (44) was excluded because of extreme findings due to the dose of atropine was prescribed according to the myopia progression rate of the patients, the treatment effect of mean annual refraction change was significantly correlated with the dose of atropine $(r=0.85 ; P$ $=0.004)$.

Heterogeneity of the meta-analysis was significant $(P<$ 0.001, $I^{2}=99 \%$; Figure 2 ). We did subgroup analysis based on different treatments in control groups (placebo or nonplacebo) and still observed significant heterogeneity in lowdose, moderate-dose, and high-dose subgroups (eFigure 2 in the Supplementary Material). In addition, a significant difference was found between Asian and white individuals in high dose atropine studies $(P<0.001)$, suggesting ethnicity might be a source of additional heterogeneity (eFigure 3 in the Supplementary Material). And this was supported by our 


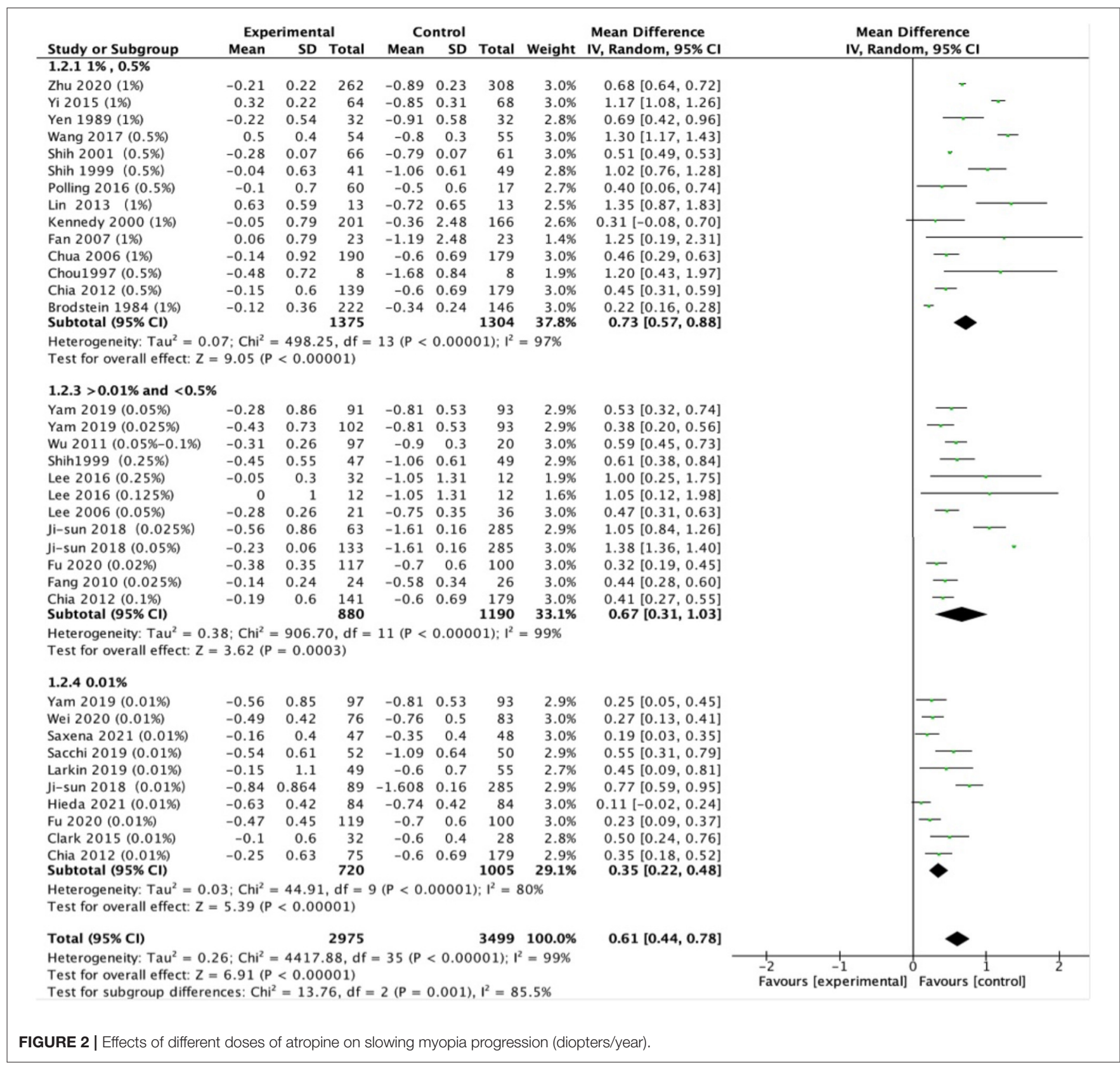

finding that there was a significant interactive effect between doses of atropine and ethnicity on mean annual refraction change (Table 2; $P$-interaction $=0.006$ ). Further analysis found that there was significant difference in refraction changes among various doses of atropine in Asian population $(P=0.008)$.

\section{Effects on Changes in Axial Length}

Thirteen studies reported changes in axial length. The analyses showed that the MD was $-0.29 \mathrm{~mm}$ in high-dose atropine studies ( $95 \% \mathrm{CI},-0.36$ to $-0.22 \mathrm{~mm} ; P<0.001$ ), $-0.23 \mathrm{~mm}$ in moderate-dose atropine studies $(95 \% \mathrm{CI},-0.27$ to $-0.18 \mathrm{~mm}$;
$P<0.001)$ and $-0.10 \mathrm{~mm}$ in low-dose atropine studies $(95 \%$ CI, -0.12 to $-0.09 \mathrm{~mm} ; P<0.001$; Figure 4). A statistically significant difference in axial elongation across various doses of atropine within this range $\left(\chi^{2}=48.81 ; P<0.001\right.$ for subgroup difference; $\left.I^{2}=95.9 \%\right)$ with significant $(P<0.001)$ heterogeneity $\left(I^{2}=99 \%\right)$. The effect sizes showed a large treatment effect for annual axial length change in different dose atropine groups (Figure 3). When the study by Moon and Shin (44) was excluded because of extreme findings, a significant dose and treatment effect on annual axial elongation was observed $(r=-0.94 ; P=$ 0.005; Figure 3). 


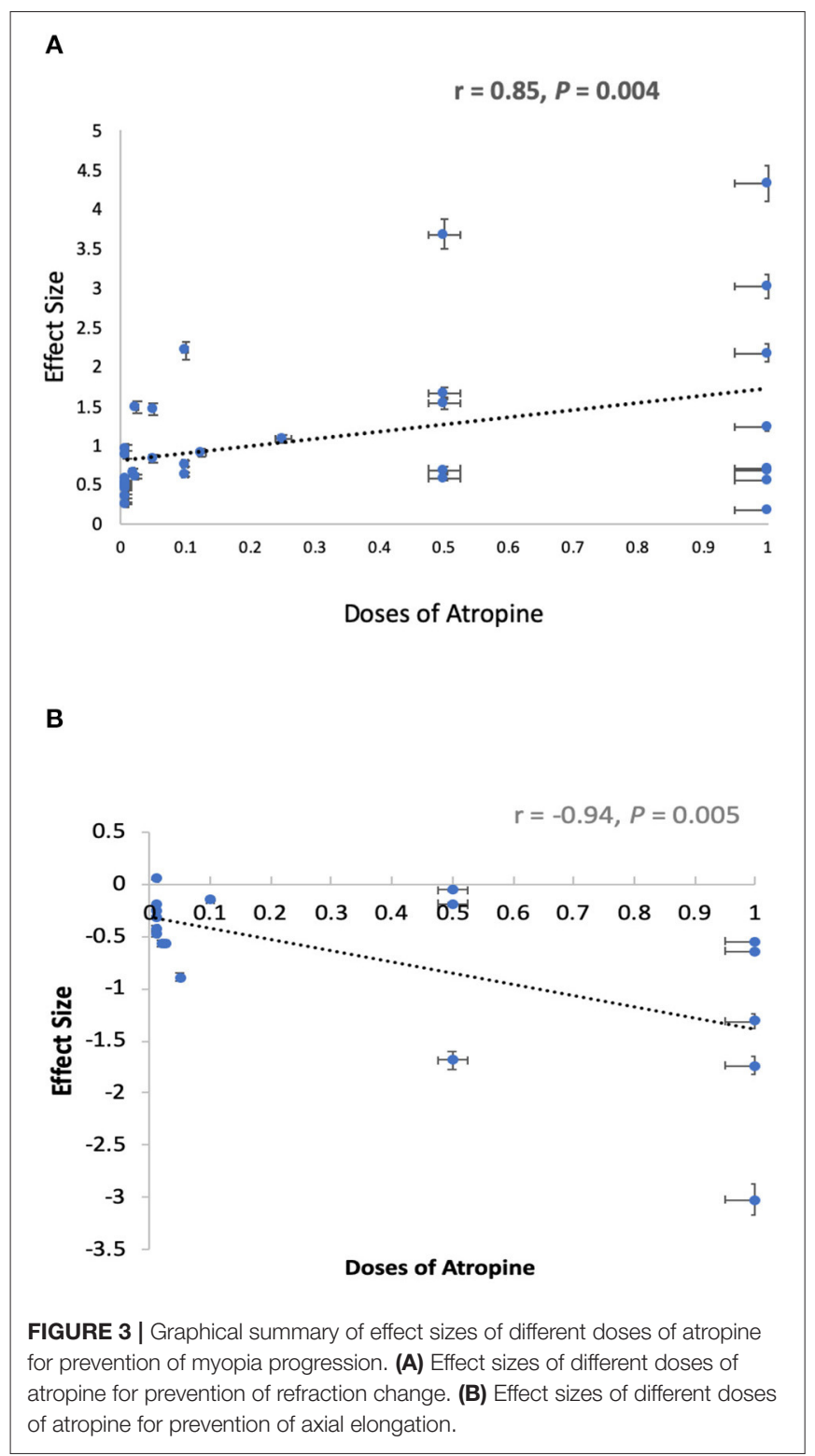

\section{Rapid Myopia Progression (>1.0 D per Year)}

Six RCTs and seven cohort studies reported the number of children with rapid myopia progression ( $>1.0 \mathrm{D}$ per year). The odds ratio (OR) of rapid myopia progression was significantly lower in atropine compared to control in both RCTs (OR, 0.13; 95\% CI, 0.10-0.18; $P<0.001)$ and cohort studies (OR, 0.19; 95\% CI, $0.10-0.3 ; P<0.001)$. The RCTs and cohort studies were combined in subsequent analyses because no difference was found between them $\left(\chi^{2}=0.93 ; P=0.33\right.$ for subgroup difference; $\left.I^{2}=0 \%\right)$. High-dose atropine showed the lowest OR for rapid myopia progression (95\% CI, 0.08-0.13; $P<0.001$ ), followed by 0.16 in moderate-dose atropine $(95 \% \mathrm{CI}, 0.08-0.31$; $\mathrm{P}<0.001)$, and 0.29 in low-dose atropine $(95 \% \mathrm{CI}, 0.18-0.47$;
TABLE 2 | Test for interaction on mean annual refraction change by doses of atropine, ethnicity, and study design.

\begin{tabular}{lccc}
\hline Characteristics & No. of studies & MD $(\mathbf{9 5 \%} \mathbf{C l})$ & $P$-interaction \\
\hline $\begin{array}{l}\text { Doses of atropine } \\
\text { High }\end{array}$ & 14 & $0.73(0.57,0.88)$ & \\
$\quad$ Moderate & 12 & $0.67(0.31,1.03)$ & \\
$\quad$ Low & 10 & $0.35(0.22,0.48)$ & \\
Ethnicity & & & $\mathbf{0 . 0 0 6}^{\star}$ \\
$\quad$ Asian patients & 21 & $0.65(0.46,0.83)$ & \\
$\quad$ White patients & 6 & $0.39(0.23,0.54)$ & \\
Study design & & $0.4508^{\dagger}$ \\
$\quad$ RCT & 18 & $0.68(0.36,1.01)$ & \\
$\quad$ Cohort studies & 19 &
\end{tabular}

MD, mean difference. Bold type indicates statistically significant. *Test for interaction between doses of atropine and ethnicity on mean annual refraction change. ${ }^{\dagger}$ Test for interaction between doses of atropine and study design on mean annual refraction change.

$P<0.001$ ) (eFigure $4 \mathrm{~A}$ in the Supplementary Material with significant difference among three groups $\left(\chi^{2}=14.88 ; P<0.001\right.$ for subgroup difference; $\left.I^{2}=86.6 \%\right)$.

\section{Slow Myopia Progression ( $<0.5$ D per Year)}

The number of children with slow myopia progression was assessed in 6 RCTs and 7 cohort studies ( $<0.5 \mathrm{D}$ per year). All of the different concentrations of atropine had a higher OR of slow myopic progression relative to control in both RCTs (OR, 6.84; 95\% CI, 4.15-11.29; $P<0.001$ ) and cohort studies (OR, 6.05; 95\% CI, 3.09-11.84; $P<0.001$ ). The combined analyses showed that the OR for atropine slowing myopia progression was 6.98 in high-dose (95\% CI, $0.08-0.13 \mathrm{~mm} ; P<0.001), 7.67$ in moderate-dose (95\% CI, 3.67-16.00; $P<0.001$ ), and 3.50 in low-dose (95\% CI, 2.02-6.06; $P<0.001$; eFigure 4B in the Supplementary Material).

\section{Treatment Efficacy With Different Treatment Durations}

Figure 5 showed the difference in efficacy of atropine between the second year and the first year. Children treated with low-dose atropine appeared to benefit more in the second year than in the first year (refraction change: $-0.23 \mathrm{D}, 95 \% \mathrm{CI},-0.39$ to -0.07 , $P=0.005$; axial elongation: $0.09 \mathrm{~mm}, 95 \% \mathrm{CI}, 0.04-0.14, P=$ $0.003)$. However, high-dose atropine showed less efficacy in the second year with a greater progression of refraction (refraction change: $0.14 \mathrm{D}, 95 \% \mathrm{CI},-0.05-0.33, P=0.14$ ) and significantly more axial elongation (axial length change: $-0.15 \mathrm{~mm}, 95 \% \mathrm{CI}$, -0.25 to $-0.05, P=0.003$ ) than in the first year of treatment (Figure 6).

\section{Side Effects}

A total of 17 studies reported the incidence of side effects. Table 3 showed the most frequently reported side effects of topical atropine, including photophobia [388 of 1,757 (25.1\%)], blurred 


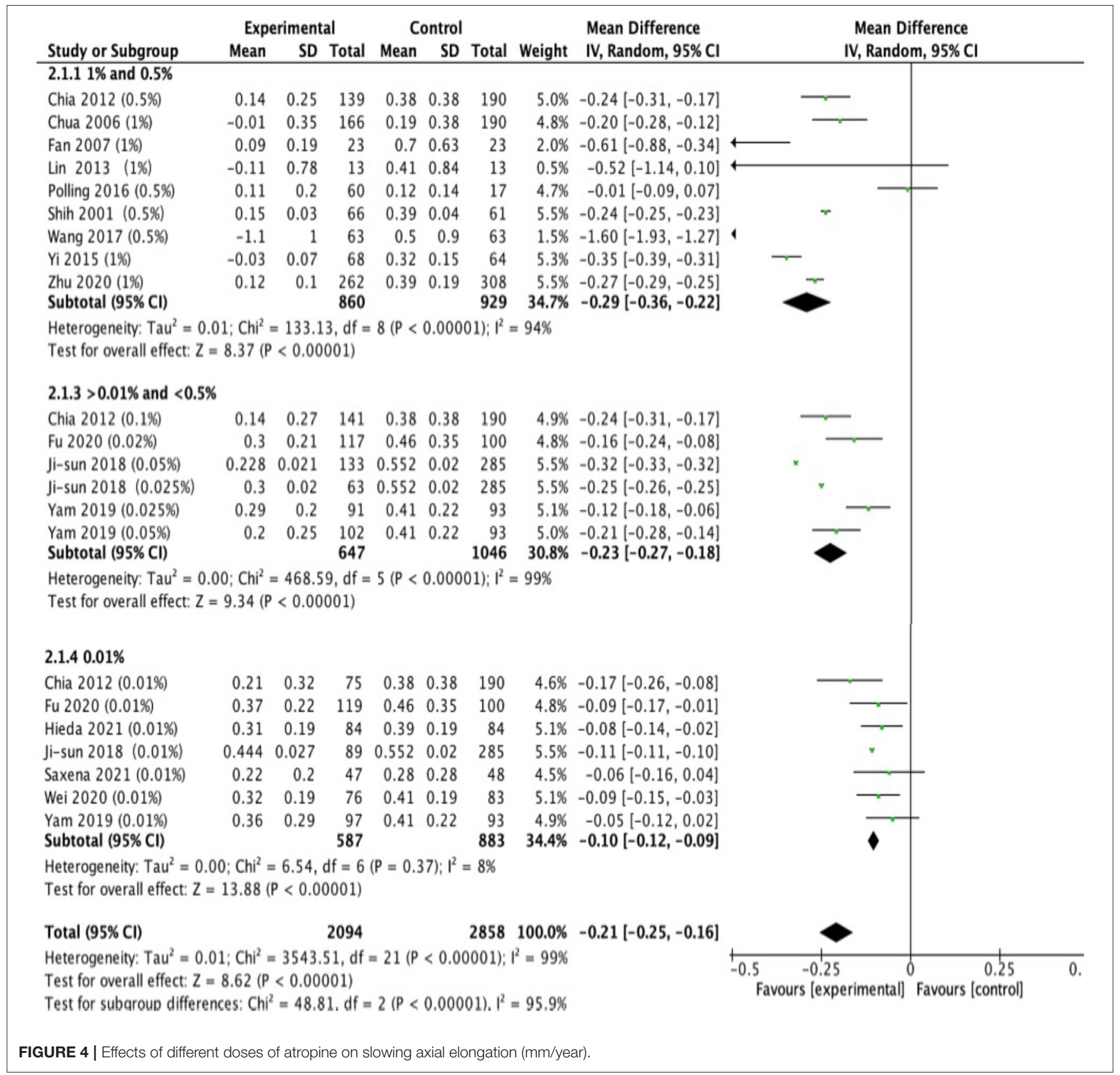

near vision [144 of $1,633(7.5 \%)$ ], and allergic reaction [49 of $1,387(2.9 \%)]$.

\section{Photophobia}

We found that all of the different concentrations of atropine had a higher OR of photophobia relative to the control $(\mathrm{OR}=16.69,95 \% \mathrm{CI}=5.37$ to 51.9 , eFigure $7 \mathrm{~A}$ in the Supplementary Material). Specifically, high-dose atropine showed the highest OR for photophobia $(\mathrm{OR}=163.57,95 \% \mathrm{CI}=$ $19.5-1,372.0)$, followed by moderate-dose atropine $(\mathrm{OR}=8.63$, $95 \% \mathrm{CI}=2.19-33.96)$, and low-dose atropine $(\mathrm{OR}=6.04,95 \%$
$\mathrm{CI}=1.39-26.23)$, showing an increase in the rate of this adverse effect with dose escalation $\left(\chi^{2}=6.83 ; P=0.03\right.$ for subgroup difference, eFigure $5 \mathrm{~A}$ in the Supplementary Material). The incidence of photophobia was statistically significant correlated with the dose of atropine $(r=0.86 ; P=0.001)$.

\section{Blurred Near Vision}

The OR for poor near visual acuity with low-, moderate- and high-dose atropine was $17.45(95 \% \mathrm{CI}=4.04-75.44), 20.52$ (95\% CI, 6.12-68.86), and 39.65 (95\% CI = 11.39-137.97), respectively (eFigure 5B in the Supplementary Material). 


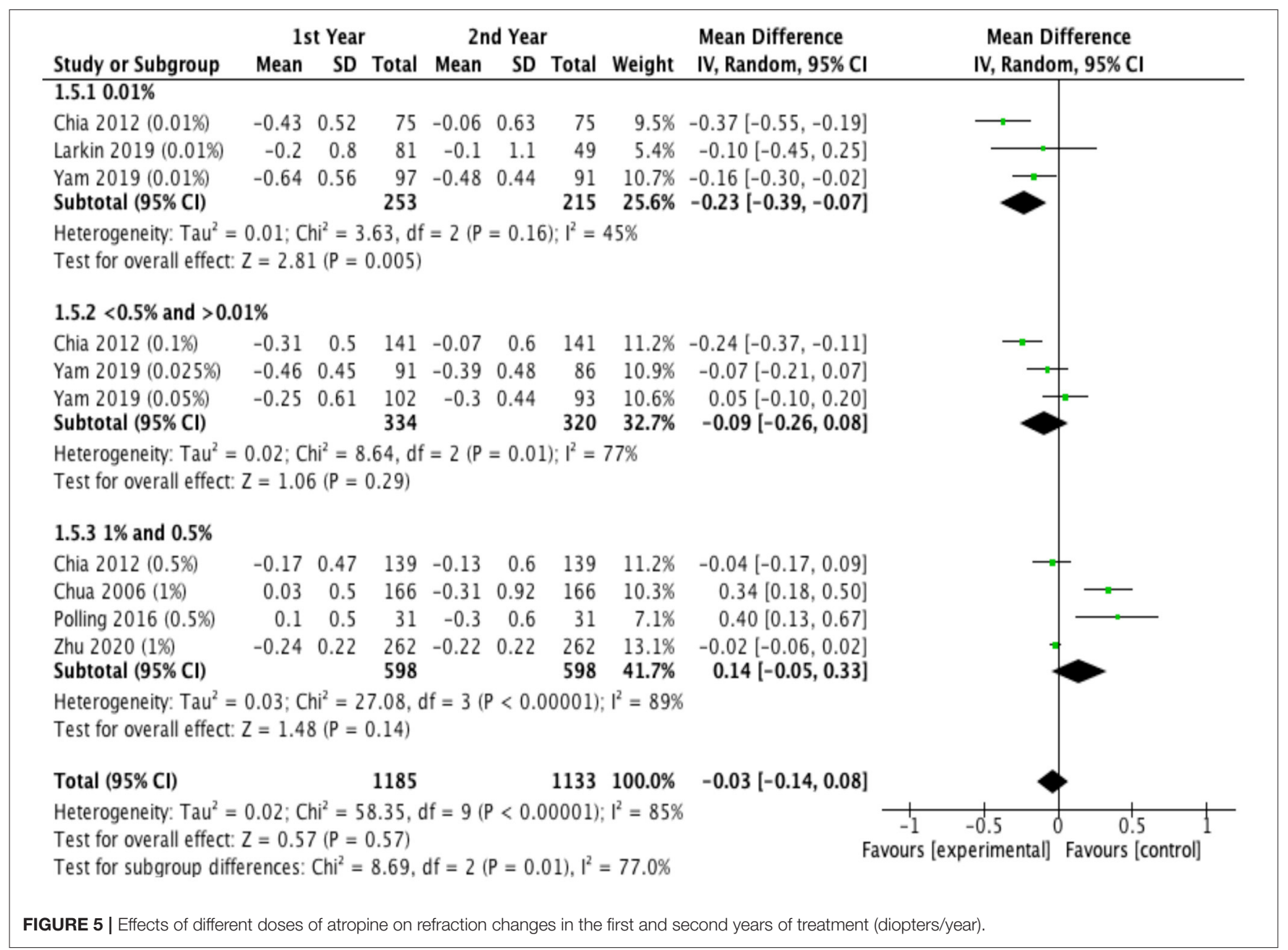

\section{Allergy}

The OR for allergies with low-, moderate, and high-dose atropine was 1.27 (95\% CI $=0.47-3.39), 1.28$ (95\% CI $=0.63-2.59)$, and 10.86 (95\% CI $=2.95-40.04$ ), respectively (eFigure $5 \mathrm{C}$ in the Supplementary Material), revealing an increase in the rate of this adverse effect with dose escalation $\left(\chi^{2}=8.68 ; P=0.01\right.$ for subgroup difference).

\section{Effects on Accommodation and Pupil Size}

We summarized the effects of atropine on accommodation amplitude in eFigure 6 in the Supplementary Material. A significant effect on accommodative amplitudes was found among groups receiving different doses of atropine, revealing a smaller decline in accommodation amplitude with low-dose atropine than with higher-dose atropine $(-1.80 \mathrm{D}$ for low-dose, $-2.7 \mathrm{D}$ for moderate-dose, and $-5.75 \mathrm{D}$ for high-dose atropine; $P<0.001)$.

As exemplified in eFigure 7 in the Supplementary Material, there was no significant difference in pupillary enlargement under photopic conditions with low-dose atropine compared with moderate-dose atropine $(P=0.91)$. Meanwhile, the number of studies examining changes in pupil size under mesopic conditions was too small (only 1 study in each subgroup) to evaluate the effect of atropine on pupil enlargement.

\section{Evaluation of the Sensitivity, Regression Analysis, and Publication Bias}

We conducted sensitivity analyses on MD in refraction change, excluding studies (1) published before 2000, (2) with baseline mean refraction $<-4 \mathrm{D}$ or (3) with a high risk of bias (eFigure 8 in the Supplementary Material). We noted that the conclusions on the outcome did not change substantially after omitting studies with significantly different characteristics. The potential sources of heterogeneity were further explored through metaregression analysis (eTable 6 in the Supplementary Material). While meta-regression analysis found ethnicity as the only statistically significant moderator with greater effects on slowing mypia progression in Asian than in white children $(0.37,95 \%$ CI 0.04-0.70).

A funnel plot for publication bias test for the outcome showed an asymmetric left-right distribution, indicating the possibility of publication bias. Factors such as insufficient sample sizes and the lack of reporting on negative results were the possible causes of publication biases (eFigure 9 in the Supplementary Material). 


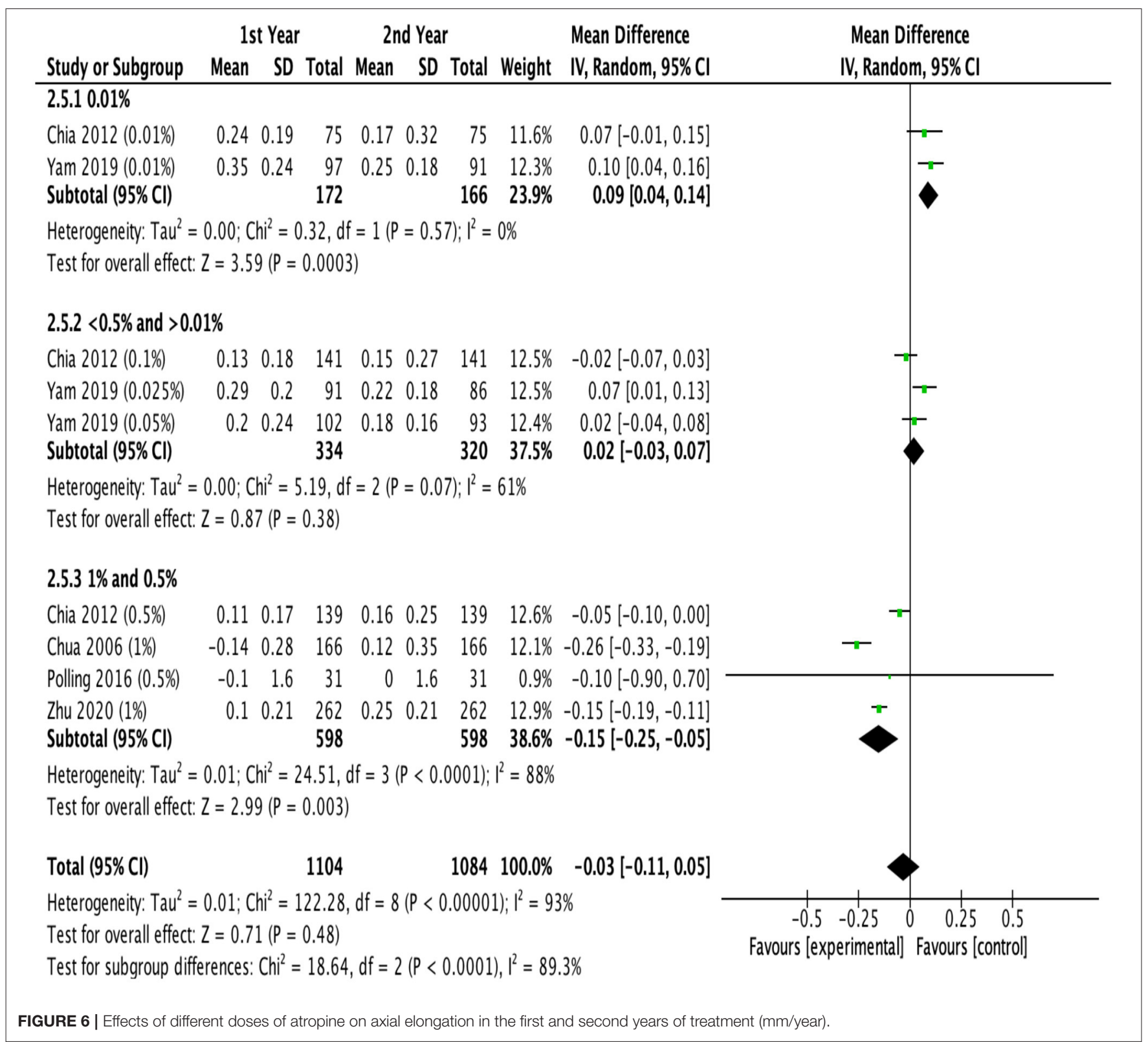

TABLE 3 | Adverse events in the atropine groups vs control group during the treatment of myopia in children.

\begin{tabular}{llccc}
\hline Outcomes & $\begin{array}{l}\text { No. of } \\
\text { studies }\end{array}$ & No. of patients & $\begin{array}{c}\text { Odds Ratio } \\
(\mathbf{9 5 \%} \text { Cl) }\end{array}$ & $\boldsymbol{I}^{\mathbf{2}}$ \\
\hline Photophobia & 5 RCTs & $\begin{array}{c}388 / 1,757 \text { vs. } \\
15 / 2,325\end{array}$ & $\begin{array}{c}16.69 \\
(5.37-51.9)\end{array}$ & $70.7 \%$ \\
& 9 Cohort & $144 / 1,633$ vs. 0 & 17.16 & 0 \\
Blurred & 4 RCTs & & $(7.97,36.95)$ & \\
near vision & 6 Cohort & & 2.24 & $77.0 \%$ \\
Allergy & 5 RCTs & $49 / 1,387$ vs. & $(1.37-3.64)$ & \\
& 2 Cohort & $21 / 1,483$ & &
\end{tabular}

\section{DISCUSSION}

In this meta-analysis, we compared the results from 12 RCTs and 15 cohort studies and confirmed that there was significantly less myopia progression $(\mathrm{MD}=0.70 \mathrm{D})$ and slower axial elongation $(\mathrm{MD}=-0.21 \mathrm{~mm})$ in the atropine group than in the control group. After excluding the study by Ji-sun et al. (43), we found that the effectiveness of atropine was related to its dose, and this was consistent with previous meta-analyses conducted in 2011 and $2020(17,55)$.

Moreover, different doses of atropine had a significantly lower $\mathrm{OR}$ in children with rapid myopia progression $(\mathrm{OR}=0.16,95 \%$ 
$\mathrm{CI}=0.11-0.23$, eFigure $4 \mathrm{~A}$ in the Supplementary Material) and a significantly higher OR in children with slow myopia progression $(\mathrm{OR}=5.88,95 \% \mathrm{CI}=3.86-8.95$, eFigure $4 \mathrm{~B}$ in the Supplementary Material), which was consistent with $\mathrm{Ha}$ et al. (18) and our previous meta-analysis published in 2014 (15).

Previous studies have demonstrated that most myopia interventions, including multifocal lenses, orthokeratology, and atropine, lost their effectiveness after the first year of treatment $(21,23,56)$. However, the review that concluded that the treatment efficacy of atropine diminished over time relied on only a single prospective study of low-dose atropine and moderatedose atropine, and therefore, the conclusion was preliminary (56). Previous studies generally presented the treatment efficacy of atropine at different time points as a cumulative effect relative to baseline. Here, we broke down the treatment efficacy into individual time segments to better illustrate the annual myopia progression during the first year and the second year of treatment. Our study suggested for the first time that the effects of low-dose atropine showed better efficacy in slowing myopia progression during the second year of treatment in protecting both refraction and axial elongation, which was consistent with the conclusion of ATOM2 study (19); moderate-dose atropine showed no difference in efficacy in the second year compared with the first year, and high-dose atropine showed less efficacy during the second year. In addition, ATOM2 study reported that compared with high-dose atropine, low-dose atropine showed the smallest rebound effect after ceasing the treatment and ended with the lowest myopic progression over the entire 3-year period (19). Therefore, low-dose atropine showed a sustained effect on inhibiting the progression of myopia in the long-term treatment. Since axial elongation naturally slows with time, it is reasonable to believe that the efficacy of high-dose atropine wanes over time. However, it is difficult to know whether the observed reductions in axial elongation with low-dose atropine during the second year were simply a function of this deceleration in growth or a change in the efficacy of atropine $(21,57)$. The treatment efficacy of atropine should be further investigated with longer followup. However, the control groups in many studies of atropine on myopia control generally given a specific dose for 1-2 years and then switched to other doses for ethical reasons, which makes long-term follow-up more difficult.

Previously, few data were available for the quantitative assessment of adverse effects of topical atropine, except the metaanalysis conducted by Gong et al. (13) and Ha et al. (18), which showed that a higher dose of atropine led to an increasing number of adverse effects. Our results also demonstrated that the side effects of atropine, such as photophobia was dosedependent. Due to the small number of reported literature on some other side effects, such as systematic symptoms (58), decline of cognitive function (59), meta-analysis cannot be done yet. Among these, systematic symptoms and decline of cognitive function have only been found in oral atropine drugs, whereas topical atropine eyedrops could hardly enter the systematic circulation by pressing the inner canthus while applying the eyedrops. And a few clinical trials have shown that children who used atropine eyedrops with 1 or 2 year follow-up periods did not show dry eye symptoms $(27,36,48)$, elevated intraocular pressure
(27), retinal photic injury $(60,61)$, though animal research found that $1 \%$ atropine eyedrops 4 times a day could induce dry eye in rabbits (62).

There have been several meta-analyses investigating various doses of atropine treatment in myopia control. A previous metaanalysis by Song et al. (55), Li et al. (15) and Gong et al. (13) included only 6, 11, and 19 studies, respectively. Recently, a network meta-analysis conducted by $\mathrm{Ha}$ et al. built up hierarchies of atropine treatment in terms of efficacy and safety among the 8 concentrations (18). But the analysis included only 16 RCTs, without comparing the treatment difference during the first year and second year.

There are some limitations in the present study. First, although this meta-analysis had established strict inclusion and exclusion criteria, the heterogeneity was still high after using the subgroup analysis. Because of insufficient data on some concentrations, different doses of atropine were combined in high dose and moderate dose studies in this meta-analysis, which might be a source of heterogeneity. And RCTs and cohort studies were combined to investigate the overall effects of different doses, although cohort studies showed similar effects to RCTs. Heterogeneity also result from ethnicity, since metaregression analysis found that atropine had greater effects on slowing mypia progression in Asian than in white children. We then conducted sensitivity analysis by omitting studies with significantly different characteristics (the year of publication year, baseline refraction, and quality of studies) and found that the outcomes remained stable. However, the publication bias analysis results showed that there might exist publication bias, so the results should be interpreted with caution. Second, more than half of the included studies did not report adverse reactions; thus, the reports on adverse effects in the included studies were not comprehensive. Third, the efficacy of atropine in our study was reported during the treatment period, and the follow-up periods significantly varied among the trials. Fourth, most of the studies evaluated were conducted among Asians. Differences between Asian and Caucasian individuals in their response to interventions for myopia progression were significant (eFigure 3, eTable 6 in the Supplementary Material). Fifth, some of the results were based on data from limited studies. For example, the effects of different doses of atropine on refraction changes in the first and second years of treatment, there were only 2 studies in some subgroups, so the results should be interpreted with caution.

Despite the limitations mentioned above, the strength of this study includes a comprehensive quantitative analysis of both efficacy and safety on varying doses of atropine. This will provide a valuable reference for the clinical application of atropine since large clinical trials for comparison of all atropine doses are unlikely to be carried out. The ideal dose of atropine in myopia control should balance efficacy and safety with the best risk/benefit ratios. In this study, low dose atropine $(0.01 \%)$ demonstrated valid efficacy in retarding refraction changes and axial elongation relative to the control group with minimal side effects and showed better efficacy in a longer follow-up period. Thus, $0.01 \%$ atropine should be advocated in the treatment for slowing myopia progression. 


\section{CONCLUSIONS}

This meta-analysis suggests that both the efficacy and the adverse effects of atropine eyedrops are dose-dependent and that the efficacy of high-dose atropine on slowing myopia progression was reduced after the first year of treatment, whereas low-dose atropine may have better efficacy in a longer follow-up period.

\section{DATA AVAILABILITY STATEMENT}

The original contributions presented in the study are included in the article/Supplementary Material, further inquiries can be directed to the corresponding author/s.

\section{AUTHOR CONTRIBUTIONS}

JG, S-ML, and NW were responsible for conceptualizing, designing, data collection, extraction, interpretation, manuscript drafting, statistical analysis, and conducting the study. JG and SWu were responsible for data collection, extraction, and critical revisions of the manuscript. KC helped on the manuscript revision. JG,

\section{REFERENCES}

1. Dolgin E. The myopia boom. Nature. (2015) 519:276-8. doi: 10.1038/519276a

2. Morgan IG, Ohno-Matsui K, Saw SM. Myopia. Lancet. (2012) 379:1739-48. doi: 10.1016/S0140-6736(12)60272-4

3. Wei SF, Sun YY, Li SM, Hu JP, Yang XH, Lin CX, et al. Refractive errors in university students in central china: the anyang university students eye study. Invest Ophthalmol Vis Sci. (2018) 59:4691-700. doi: 10.1167/iovs.18-24363

4. Li S, Li S, Kang M, Zhou Y, Li H, Liu L, et al. Distribution of ocular biometry in 7- and 14-year-old Chinese children. Optom Vis Sci. (2015) 92:566-72. doi: 10.1097/OPX.0000000000000570

5. Baird P, Saw S, Lanca C, Guggenheim J, Smith Iii E, Zhou X, et al. Myopia. Nat Rev Dis Primers. (2020) 6:99. doi: 10.1038/s41572-020-00231-4

6. Lin L, Shih Y, Hsiao C, Chen C. Prevalence of myopia in Taiwanese schoolchildren: 1983 to 2000. Ann Acad Med Singap. (2004) 33:27-33.

7. Wang J, Li Y, Musch D, Wei N, Qi X, Ding G, et al. Progression of myopia in school-aged children after COVID-19 home confinement. JAMA Ophthalmol. (2021) 139:293-300. doi: 10.1001/jamaophthalmol.2020.6239

8. Li S, Li H, Li S, Liu L, Kang M, Wang Y, et al. Time outdoors and myopia progression over 2 years in chinese children: the anyang childhood eye study. Invest Ophthalmol Vis Sci. (2015) 56:4734-40. doi: 10.1167/iovs.14-15474

9. He M, Xiang F, Zeng Y, Mai J, Chen Q, Zhang J, et al. Effect of time spent outdoors at school on the development of myopia among children in china: a randomized clinical trial. JAMA. (2015) 314:1142-8. doi: 10.1001/jama.2015.10803

10. Wu P, Chen C, Lin K, Sun C, Kuo C, Huang H, et al. Myopia prevention and outdoor light intensity in a school-based cluster randomized trial. Ophthalmology. (2018) 125:1239-50. doi: 10.1016/j.ophtha.2017.12.011

11. Walline J, Lindsley K, Vedula S, Cotter S, Mutti D, Ng S, et al. Interventions to slow progression of myopia in children. Cochrane Database Syst Rev. (2020) 1:CD004916. doi: 10.1002/14651858.CD004916.pub4

12. Fang Y, Chou Y, Pu C, Lin P, Liu T, Huang N, et al. Prescription of atropine eye drops among children diagnosed with myopia in Taiwan from 2000 to 2007: a nationwide study. Eye. (2013) 27:418-24. doi: 10.1038/eye.2012.279

13. Gong Q, Janowski M, Luo M, Wei H, Chen B, Yang G, et al. Efficacy and adverse effects of atropine in childhood myopia a meta-analysis. JAMA Ophthalmol. (2017) 135:624-30. doi: 10.1001/jamaophthalmol.2017. 1091

14. Chia A, Chua WH, Wen L, Fong A, Goon YY, Tan D. Atropine for the treatment of childhood myopia: changes after stopping atropine
$\mathrm{DM}, \mathrm{XH}, \mathrm{ZH}, \mathrm{M}-\mathrm{TK}, \mathrm{SWe}$, and $\mathrm{WB}$ were responsible for data interpretation, manuscript drafting, supervision, and critical revisions of the manuscript for important intellectual content. S-ML and $\mathrm{NW}$ are the guarantors of this article and takes full responsibility for this study. All authors contributed to the article and approved the submitted version.

\section{FUNDING}

This works was supported by grants from the Capital Health Research and Development of Special (2020-2-1081), Beijing Natural Science Foundation (JQ20029), Beijing Talents Found (2016000021223ZK28), and the National Natural Science Foundation of China (82071000).

\section{SUPPLEMENTARY MATERIAL}

The Supplementary Material for this article can be found online at: https://www.frontiersin.org/articles/10.3389/fmed. 2021.756398/full\#supplementary-material
$0.01 \%, \quad 0.1 \%$ and $0.5 \%$. Am J Ophthalmol. (2014) 157:451-7.e1. doi: 10.1016/j.ajo.2013.09.020

15. Li SM, Wu SS, Kang MT, Liu Y, Jia SM, Li SY, et al. Atropine slows myopia progression more in Asian than white children by meta-analysis. Optom Vis Sci. (2014) 91:342-50. doi: 10.1097/OPX.0000000000000178

16. Wei S, Li S, An W, Du J, Liang X, Sun Y, et al. Safety and efficacy of lowdose atropine eyedrops for the treatment of myopia progression in chinese children: a randomized clinical trial. JAMA Ophthalmol. (2020) 138:1178-84. doi: 10.1001/jamaophthalmol.2020.3820

17. Zhao C, Cai C, Ding Q, Dai H. Efficacy and safety of atropine to control myopia progression: a systematic review and meta-analysis. BMC Ophthalmol. (2020) 20:478. doi: 10.1186/s12886-020-01746-w

18. Ha A, Kim S, Shim S, Kim Y, Jung J. Efficacy and safety of 8 atropine concentrations for myopia control in children: a network meta-analysis. Ophthalmology. (2021) doi: 10.1016/j.ophtha.2021.10.016

19. Chia A, Lu QS, Tan D. Five-Year clinical trial on atropine for the treatment of myopia 2: myopia control with atropine $0.01 \%$ eyedrops. Ophthalmology. (2016) 123:391-9. doi: 10.1016/j.ophtha.2015.07.004

20. Sankaridurg P, Tran H. The lowdown on low-concentration atropine for myopia progression. Ophthalmology. (2019) 126:125-6. doi: 10.1016/j.ophtha.2018.08.024

21. Brennan N, Toubouti Y, Cheng X, Bullimore M. Efficacy in myopia control. Prog Retin Eye Res. (2020) 83:100923. doi: 10.1016/j.preteyeres.2020.100923

22. Hutton B, Salanti G, Caldwell D, Chaimani A, Schmid C, Cameron C, et al. The PRISMA extension statement for reporting of systematic reviews incorporating network meta-analyses of health care interventions: checklist and explanations. Ann Intern Med. (2015) 162:777-84. doi: 10.7326/M14-2385

23. Huang J, Wen D, Wang Q, McAlinden C, Flitcroft I, Saw SM, et al. Efficacy comparison of 16 interventions for myopia control in children: a network meta-analysis. Ophthalmology. (2016) 123:697-708. doi: 10.1016/j.ophtha.2015.11.010

24. Higgins J, Altman D, Gøtzsche P, Jüni P, Moher D, Oxman A, et al. The cochrane collaboration's tool for assessing risk of bias in randomised trials. BMJ. (2011) 343:d5928. doi: 10.1136/bmj.d5928

25. Wells G. The newcastle-ottawa scale (NOS) for assessing the quality of non randomized studies in meta-analyses. In: Symposium on Systematic Reviews: Beyond the Basics Ottawa (2014). Ottawa.

26. Stang A. Critical evaluation of the newcastle-ottawa scale for the assessment of the quality of nonrandomized studies in meta-analyses. Eur J Epidemiology. (2010) 25:603-5. doi: 10.1007/s10654-010-9491-z 
27. Chua W-H, Balakrishnan V, Chan Y-H, Tong L, Ling Y, Quah B-L, et al. Atropine for the treatment of childhood myopia. Ophthalmology. (2006) 113:2285-91. doi: 10.1016/j.ophtha.2006.05.062

28. Chia A, Chua W-H, Cheung Y-B, Wong W-L, Lingham A, Fong A, et al. Atropine for the treatment of childhood myopia: safety and efficacy of $0.5 \%, 0.1 \%$, and $0.01 \%$ doses (Atropine for the treatment of myopia 2). Ophthalmology. (2012) 119:347-54. doi: 10.1016/j.ophtha.2011. 07.031

29. Liang CK, Ho TY, Li TC, Hsu WM, Li TM, Lee YC, et al. A combined therapy using stimulating auricular acupoints enhances lower-level atropine eyedrops when used for myopia control in school-aged children evaluated by a pilot randomized controlled clinical trial. Complement Ther Med. (2008) 16:305-10. doi: 10.1016/j.ctim.2008.04.007

30. Shih YF, Chen CH, Chou AC, Ho TC, Lin LL, Hung PT. Effects of different concentrations of atropine on controlling myopia in myopic children. J Ocul Pharmacol Ther. (1999) 15:85-90. doi: 10.1089/jop.1999.15.85

31. Shih YF, Hsiao CK, Chen CJ, Chang CW, Hung PT, Lin LL. An intervention trial on efficacy of atropine and multi-focal glasses in controlling myopic progression. Acta Ophthalmol Scand. (2001) 79:233-6. doi: 10.1034/j.1600-0420.2001.790304.x

32. Wang Y-R, Bian H-L, Wang Q. Atropine 0.5\% eyedrops for the treatment of children with low myopia: a randomized controlled trial. Medicine. (2017) 96:e7371. doi: 10.1097/MD.0000000000007371

33. Zhu Q, Tang Y, Guo L, Tighe S, Zhou Y, Zhang X, et al. Efficacy and safety of $1 \%$ atropine on retardation of moderate myopia progression in chinese school children. Int J Med Sci. (2020) 17:176-81. doi: 10.7150/ijms. 39365

34. Yam JC, Jiang Y, Tang SM, Law AKP, Chan JJ, Wong E, et al. LowConcentration atropine for myopia progression (LAMP) study: a randomized, double-blinded, placebo-controlled trial of $0.05 \%, 0.025 \%$, and $0.01 \%$ atropine eye drops in myopia control. Ophthalmology. (2019) 126:113-24. doi: 10.1016/j.ophtha.2018.05.029

35. Yen MY, Liu JH, Kao SC, Shiao CH. Comparison of the effect of atropine and cyclopentolate on myopia. Ann Ophthalmol. (1989) 21:180-7.

36. Yi S, Huang Y, Yu S-Z, Chen X-J, Yi H, Zeng X-L. Therapeutic effect of atropine $1 \%$ in children with low myopia. J AAPOS. (2015) 19:426-9. doi: 10.1016/j.jaapos.2015.04.006

37. Bedrossian RH. The treatment of myopia with atropine and bifocals: a long-term prospective study. Ophthalmology. (1985) 92:716. doi: 10.1016/S0161-6420(85)33974-X

38. Chou AC, Shih YF, Ho TC, Lin LL. The effectiveness of $0.5 \%$ atropine in controlling high myopia in children. J Ocul Pharmacol Ther. (1997) 13:61-7. doi: 10.1089/jop.1997.13.61

39. Fan DS, Lam DS, Chan CK, Fan AH, Cheung EY, Rao SK. Topical atropine in retarding myopic progression and axial length growth in children with moderate to severe myopia: a pilot study. Jpn J Ophthalmol. (2007) 51:27-33. doi: 10.1007/s10384-006-0380-7

40. Lee J-J, Fang P-C, Yang IH, Chen C-H, Lin P-W, Lin S-A, et al. Prevention of myopia progression with $0.05 \%$ atropine solution. J Ocul Pharmacol Ther. (2006) 22:41-6. doi: 10.1089/jop.2006.22.41

41. Lin L, Lan W, Liao Y, Zhao F, Chen C, Yang Z. Treatment outcomes of myopic anisometropia with 1\% atropine: a pilot study. Optom Vis Sci. (2013) 90:1486. doi: 10.1097/OPX.0000000000000097

42. Clark TY, Clark RA. Atropine $0.01 \%$ eyedrops significantly reduce the progression of childhood myopia. J Ocul Pharmacol Ther. (2015) 31:541-545. doi: 10.1089/jop.2015.0043

43. Fang P-C, Chung M-Y, Yu H-J, Wu P-C. Prevention of myopia onset with $0.025 \%$ atropine in premyopic children. J Ocul Pharmacol Ther. (2010) 26:341-5. doi: 10.1089/jop.2009.0135

44. Moon JS, Shin SY. The diluted atropine for inhibition of myopia progression in Korean children. Int J Ophthalmol. (2018) 11:1657-62.

45. Kennedy RH, Dyer JA, Kennedy MA, Parulkar S, Kurland LT, Herman DC, et al. Reducing the progression of myopia with atropine: a long term cohort study of olmsted County students. Binocul Vis Strabismus Q. (2000) 15:281304.

46. Lee CY, Sun CC, Lin YF, Lin KK. Effects of topical atropine on intraocular pressure and myopia progression: a prospective comparative study. BMC Ophthalmol. (2016) 16:114. doi: 10.1186/s12886-016-0297-y
47. Larkin G, Luke, Tahir A, David EK, Beauchamp C, Tong J, et al. Atropine $0.01 \%$ eye drops for myopia control in american children: a multiethnic sample across three US sites. Ophthalmol Ther. (2019) 8:589-98. doi: 10.1007/s40123-019-00217-w

48. Polling JR, Kok RG, Tideman JW, Meskat B, Klaver CC. Effectiveness study of atropine for progressive myopia in Europeans. Eye. (2016) 30:998-1004. doi: 10.1038 /eye.2016.78

49. Sacchi M, Serafino M, Villani E, Tagliabue E, Luccarelli S, Bonsignore F, et al. Efficacy of atropine $0.01 \%$ for the treatment of childhood myopia in European patients. Acta Ophthalmol. (2019) 97:e1136-40. doi: 10.1111/aos.14166

50. Wu P-C, Yang Y-H, Fang P-C. The long-term results of using low-concentration atropine eye drops for controlling myopia progression in schoolchildren. J Ocul Pharmacol Ther. (2011) 27:461-6. doi: 10.1089/jop.2011.0027

51. Fu A, Stapleton F, Wei L, Wang W, Zhao B, Watt K, et al. Effect of lowdose atropine on myopia progression, pupil diameter and accommodative amplitude: low-dose atropine and myopia progression. $\mathrm{Br} J$ Ophthalmol. (2020) 104:1535-41. doi: 10.1136/bjophthalmol-2019-315440

52. Saxena R, Dhiman R, Gupta V, Kumar P, Matalia J, Roy L, et al. Atropine for treatment of childhood myopia in India (I-ATOM): multicentric randomized trial. Ophthalmology. (2021) 128:1367-9. doi: 10.1016/j.ophtha.2021.01.026

53. Hieda O, Hiraoka T, Fujikado T, Ishiko S, Hasebe S, Torii H, et al. Efficacy and safety of $0.01 \%$ atropine for prevention of childhood myopia in a 2-year randomized placebo-controlled study. Jpn J Ophthalmol. (2021) 65:315-25. doi: 10.1007/s10384-021-00822-y

54. Polling J, Tan E, Driessen S, Loudon S, Wong H, Astrid S, et al. A 3-year follow-up study of atropine treatment for progressive myopia in Europeans. Eye. (2020) 34:2020-8. doi: 10.1038/s41433-020-1122-7

55. Song YY, Wang H, Wang B-S, Qi H, Rong Z-X, Chen H-Z. Atropine in ameliorating the progression of myopia in children with mild to moderate myopia: a meta-analysis of controlled clinical trials. J Ocul Pharmacol Ther. (2011) 27:361-8. doi: 10.1089/jop.2011.0017

56. Brennan NA, Cheng X. Commonly held beliefs about myopia that lack a robust evidence base. Eye Contact Lens. (2019) 45:215-25. doi: 10.1097/ICL.0000000000000566

57. Matsumura S, Lanca C, Htoon H, Brennan N, Tan C, Kathrani B, et al. Annual myopia progression and subsequent 2-year myopia progression in Singaporean children. Transl Vis Sci Technol. (2020) 9:12. doi: $10.1167 /$ tvst.9.13.12

58. Princelle A, Hue V, Pruvost I, Potey C, Dubos F, Martinot A. Systemic adverse effects of topical ocular instillation of atropine in two children. Arch Pediatr. (2013) 20:391-4. doi: 10.1016/j.arcped.2013.01.012

59. Pasina L, Djade C, Lucca U, Nobili A, Tettamanti M, Franchi C, et al. Association of anticholinergic burden with cognitive and functional status in a cohort of hospitalized elderly: comparison of the anticholinergic cognitive burden scale and anticholinergic risk scale: results from the REPOSI study. Drugs Aging. (2013) 30:103-12. doi: 10.1007/s40266-012-0044-x

60. Chia A, Li W, Tan D, Luu CD. Full-field electroretinogram findings in children in the atropine treatment for myopia (ATOM2) study. Document Ophthalmol. (2013) 126:177-86. doi: 10.1007/s10633-012-9372-8

61. Luu CD, Lau AM, Koh AH, Tan D. Multifocal electroretinogram in children on atropine treatment for myopia. Br J Ophthalmol. (2005) 89:151-3. doi: 10.1136/bjo.2004.045526

62. Shi W, Li Q, Wang Y, Huang J, Zhang C. Establishment of a rabbit dry eye model by using sulfate atropine eye drops. J Clin Ophthalmol. (2011) 19:455-57. doi: 10.3969/j.issn.1006-8422.2011.05.030

Author Disclaimer: This article's contents are solely the responsibility of the authors.

Conflict of Interest: The authors declare that the research was conducted in the absence of any commercial or financial relationships that could be construed as a potential conflict of interest.

Publisher's Note: All claims expressed in this article are solely those of the authors and do not necessarily represent those of their affiliated organizations, or those of the publisher, the editors and the reviewers. Any product that may be evaluated in 
this article, or claim that may be made by its manufacturer, is not guaranteed or endorsed by the publisher.

Copyright $\odot 2022$ Gan, $L i, W u, C a o, M a, H e, H u a$, Kang, Wei, Bai and Wang. This is an open-access article distributed under the terms of the Creative Commons
Attribution License (CC BY). The use, distribution or reproduction in other forums is permitted, provided the original author(s) and the copyright owner(s) are credited and that the original publication in this journal is cited, in accordance with accepted academic practice. No use, distribution or reproduction is permitted which does not comply with these terms. 